\title{
Rehabilitation following hip arthroscopy - a systematic review
}

\author{
Jeffrey S. Grzybowski ${ }^{1}$, Philip Malloy ${ }^{2}$, Catherine Stegemann ${ }^{1}$, Charles Bush-Joseph ${ }^{1}$, \\ Joshua David Harris ${ }^{3 *}$ and Shane J. Nho ${ }^{1}$
}

${ }^{1}$ Hip Preservation Center, Department of Orthopedic Surgery, Rush University Medical Center, Chicago, IL, USA, ${ }^{2}$ Department of Physical Therapy, Marquette University College of Health Sciences, Milwaukee, WI, USA, ${ }^{3}$ Houston Methodist Orthopedics and Sports Medicine, Houston, TX, USA

Context: Rehabilitation following hip arthroscopy is an integral component of the clinical outcome of the procedure. Given the increase in quantity, complexity, and diversity of procedures performed, a need exists to define the role of rehabilitation following hip arthroscopy.

Objectives: (1) To determine the current rehabilitation protocols utilized following hip arthroscopy in the current literature, (2) to determine if clinical outcomes are significantly

OPEN ACCESS

Edited by:

Vassilios S. Nikolaou, University of Athens, Greece

Reviewed by:

Konstantinos Markatos, University of Athens, Greece Narayan Hulse,

Fortis Hospital, India Athanasios Papavasiliou, Aristotle University of Thessaloniki,

Greece

*Correspondence:

Joshua David Harris, 6550 Fannin Street, Smith Tower,

Suite 2500, Houston, TX, USA joshuaharrismd@gmail.com

Specialty section:

This article was submitted to Orthopedic Surgery, a section of the journal Frontiers in Surgery

Received: 08 April 2015

Accepted: 10 May 2015

Published: 26 May 2015

Citation:

Grzybowski JS, Malloy $P$, Stegemann C, Bush-Joseph C,

Harris JD and Nho SJ (2015)

Rehabilitation following hip arthroscopy - a systematic review.

Front. Surg. 2:21.

doi: 10.3389/fsurg.2015.00021 different based on different post-operative rehabilitation protocols, and (3) to propose the best-available evidence-based rehabilitation program following hip arthroscopy.

Data sources: Per PRISMA guidelines and checklist, Medline, SciVerse Scopus, SportDiscus, and Cochrane Central Register of Controlled Trials were searched.

Study selection: Level I-IV evidence clinical studies with minimum 2-year followup reporting outcomes of hip arthroscopy with post-operative rehabilitation protocols described were included.

Data extraction: All study, subject, and surgery parameters were collected. All elements of rehabilitation were extracted and analyzed. Descriptive statistics were calculated. Study methodological quality was analyzed using the modified Coleman methodology score.

Results: Eighteen studies were included (2,092 subjects; $52 \%$ male, mean age $35.1 \pm 10.6$ years, mean follow-up $3.2 \pm 1.0$ years). Labral tear and femoroacetabular impingement were the most common diagnoses treated and labral debridement and femoral/acetabular osteochondroplasty the most common surgical techniques performed. Rehabilitation protocol parameters (weight-bearing, motion, strengthening, and return to sport) were poorly reported. Differences in clinical outcomes were unable to be assessed given heterogeneity in study reporting. Time-, phase-, goal-, and precautionbased guidelines were extracted and reported.

Conclusion: The current literature of hip arthroscopy rehabilitation lacks high-quality evidence to support a specific protocol. Heterogeneity in study, subject, and surgical demographics precluded assimilation of protocols and/or outcomes to generate evidencebased guidelines. Strengths and limitations in the literature were identified. Future studies should recognize and report the essentials of rehabilitation following hip arthroscopy.

Keywords: hip, arthroscopy, rehabilitation, physical therapy 


\section{Introduction}

Femoroacetabular impingement (FAI) is a common cause of pain that may lead to osteoarthritis of the hip (1). Cam and pincer FAI are two distinct anatomic entities that may lead to abnormal articular congruity and subsequent chondrolabral dysfunction (1). The acetabular labrum is an important structure in hip preservation based on improved surgical outcomes after repair vs. debridement during FAI surgery (femoral osteochondroplasty and acetabular rim trimming) (2). Early- and mid-term follow-up after FAI surgery has revealed significant improvements in hip-specific (3), general health-specific (4), and quality of life (4) questionnaires. Nevertheless, it is unknown whether FAI surgery and labral repair may prevent long-term degenerative changes of the hip (5). In addition to FAI and labral tears, several other intra- and extraarticular causes of hip pain may warrant arthroscopic/endoscopic treatment including synovial chondromatosis, loose bodies, snapping iliopsoas or iliotibial band, ligamentum teres tear, hip abductor tears, trochanteric bursitis, and proximal hamstring tear.

Rehabilitation following hip arthroscopy has long been recognized as an integral component of the clinical outcome of the procedure (6). The wide variety of bony and soft-tissue procedures precludes a standard post-operative rehabilitation for "hip arthroscopy." Over the past decade, the incidence of hip arthroscopy has risen dramatically (7). Given the increase in quantity, complexity, and diversity of procedures performed, a need exists to define the role of rehabilitation following hip arthroscopy. The purposes of this systematic review are (1) to determine the current rehabilitation protocols utilized following hip arthroscopy in the current literature, (2) to determine if clinical outcomes are significantly different based on different post-operative rehabilitation protocols, and (3) to propose the best-available evidence-based rehabilitation program following hip arthroscopy. The authors hypothesize that (1) post-operative rehabilitation protocols are infrequently and poorly reported with significant heterogeneity, and (2) there is little to no evidence that supports or refutes specific post-operative rehabilitation protocols and that current protocols are based on theory and biomechanical, rather than clinical, investigations.

\section{Methods}

A systematic review was conducted according to preferred reporting items for systematic reviews and meta-analyses (PRISMA) guidelines using a PRISMA checklist (8). Systematic review registration was performed using the PROSPERO International prospective register of systematic reviews (registration number CRD42013003760) (9). Two reviewers conducted the search separately on January 31, 2013 using the following databases: Medline, SportDiscus, CINAHL, and PEDro. A specific electronic search citation algorithm was utilized ${ }^{1}$. English language Level I-IV

${ }^{1}((((()((()((()(((($ arthroscopy[Title/Abstract]) $)$ AND hip[Title/Abstract])) NOT shoulder[Title/Abstract]) NOT elbow[Title/Abstract]) NOT wrist[Title/Abstract]) NOT knee[Title/Abstract]) NOT ankle[Title/Abstract])) NOT lumbar[Title/ Abstract]) NOT lumbosacral[Title/Abstract]) NOT sacrum[Title/Abstract]) NOT sacroiliac[Title/Abstract]) NOT sacral[Title/Abstract])) NOT cadaver[Title/Abstract]) NOT cadaveric[Title/Abstract]) NOT biomechanical[Title/Abstract])) NOT revision[Title] AND (English[lang]). evidence [2011 update by the Oxford Centre for Evidence-Based Medicine (10)] clinical outcome studies with minimum 2-year follow-up were eligible. Medical conference abstracts were ineligible for inclusion. All references within included studies were cross-referenced for inclusion if missed by the initial search. Duplicate subject publications within separate unique studies were not reported twice. The studies with longer duration followup, greater number of subjects, or more explicit reporting of rehabilitation were retained for inclusion. Level $\mathrm{V}$ evidence reviews, letters to the editor, basic science, biomechanical studies, open hip surgery, imaging, surgical technique, and classification studies were excluded. Inclusive studies necessarily reported post-operative rehabilitation protocols. Qualitative and quantitative reporting of specific rehabilitation parameters was analyzed. Those studies that otherwise would have been eligible for inclusion and analysis (e.g., 2 years clinical follow-up after hip arthroscopy) that failed to include any post-operative rehabilitation protocol were excluded.

Subjects of interest in this systematic review were enrolled in a clinical trial with a minimum of 2 years follow-up following hip arthroscopy (intervention). Specific outcomes of interest regarding post-operative rehabilitation included weight-bearing status, motion, continuous passive motion (CPM), stationary bike, crutches, brace, anti-rotation boots, heterotopic ossification (HO) prophylaxis, and return to sport. Specific surgical outcomes of interest included intra- and extra-articular procedures including arthroscopic femoral osteochondroplasty, pincer acetabuloplasty, labral debridement or repair, loose body removal, articular cartilage surgery, capsular repair/plication or release, iliopsoas release, ligamentum teres debridement, gluteus medius/minimus debridement or repair, iliotibial release or windowing, and greater trochanteric bursectomy. Study and subject demographic parameters analyzed included year of publication, years of subject enrollment, presence of study financial conflict of interest, number of subjects and hips, gender, age, body mass index (BMI), diagnoses treated, and surgical procedures performed. Clinical outcome scores sought were the non-arthritic hip score (NAHS), international Hip Outcome Tool-12 (iHOT-12), hip outcome score (HOS), modified Harris hip score (mHHS), and hip disability and osteoarthritis outcome score (HOOS). Study methodological quality was evaluated using the modified Coleman methodology score (MCMS) (11). The authors declare that no financial conflict of interest influenced the topic of this manuscript.

Study descriptive statistics were calculated. Continuous variable data were reported as mean \pm SD from the mean. Categorical variable data were reported as frequency with percentages. For all statistical analysis either measured and calculated from study data extraction or directly reported from the individual studies, $p<0.05$ was considered statistically significant.

\section{Results}

\section{Study, Subject, and Surgical Demographics}

Eighteen studies were identified for analysis (Figure 1) (3, 4, 12-27). Eligible subjects were enrolled between 1992 and 2010. Eight studies (44\%) denied and five studies (28\%) reported the presence of a financial conflict of interest, while five studies (28\%) did not report the presence or absence of a financial conflict 


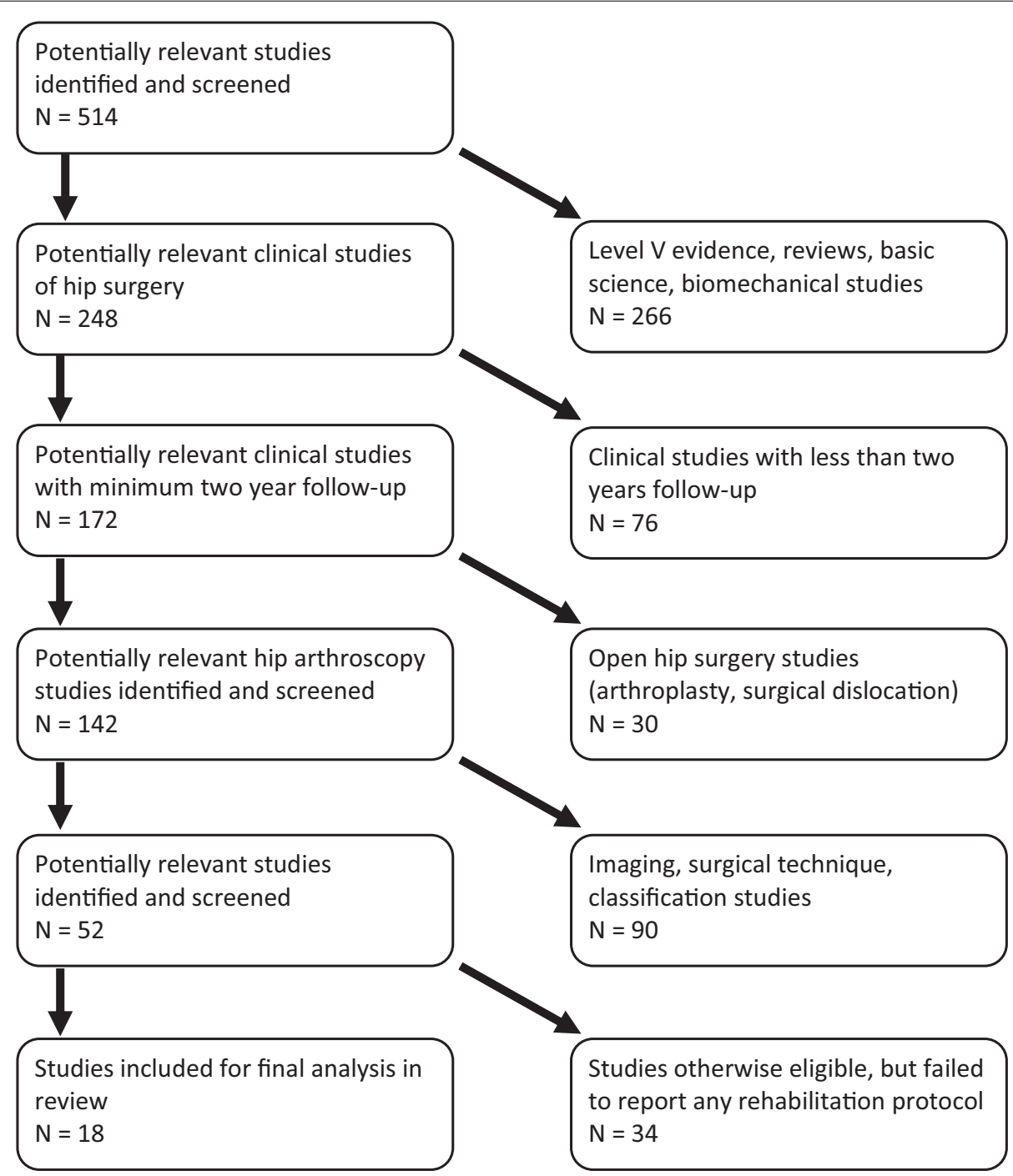

FIGURE 1 | PRISMA flowchart for selection of included and analyzed studies

of interest. Fifteen studies (83\%) were Level IV evidence, two (11\%) were Level III, and one (6\%) was Level I evidence. There were 2,092 subjects (2,099 hips) analyzed with 52\% male (48\% female), $48 \%$ right ( $52 \%$ left) hips, with mean age $35.1 \pm 10.6$ years (range 16.9-56.5 years) and mean BMI $24.3 \pm 2.4 \mathrm{~kg} / \mathrm{m}^{2}$. When present, the mean time from symptom presentation to surgery was $23.1 \pm 15$ months. Sixty-seven percent of surgeries $(n=1,408$ subjects) were performed in supine position (33\% lateral; $n=691$ subjects). Mean length of follow-up was $3.2 \pm 1.0$ years.

Fifty-four percent $(n=1,127)$ and $80 \%(n=1,676)$ of hips were diagnosed with FAI and labral tears, respectively. When reported, 67\% $(n=634), 5.5 \%(n=52)$, and $28 \%(n=28 \%)$ were diagnosed with cam, pincer, and mixed FAI, respectively. Other primary diagnoses treated were osteoarthritis (35\% of all hips; $n=744)$, ligamentum teres tear $(27 \% ; n=568)$, chondral defects of acetabulum, femur, or both $(16 \% ; n=330)$, loose bodies or synovial chondromatosis $(5 \% ; n=98)$, and iliopsoas tendon pathology $(3 \% ; n=62)$. Labral debridement was the most common surgical technique performed $(66 \% ; n=1,383)$, followed by femoral osteochondroplasty $(52 \% ; n=1,093)$, ligamentum teres debridement $(29 \% ; n=599)$, acetabuloplasty rim trimming $(17 \% ; n=355)$, labral repair $(16 \% ; n=346)$, microfracture of femoral head and/or acetabulum $(9 \% ; n=186)$, loose body removal $(5 \% ; n=115)$, and iliopsoas release $(3 \% ; n=62)$.

Mean MCMS was $33.8 \pm 9.6$ (poor quality). Study strengths (via MCMS) were length of follow-up, treatment description, and description of rehabilitation protocol. Study limitations were blinding, randomization, number of patients needed to treat analysis, and power analysis and alpha error calculations. MCMS question 13 (description of rehab protocol - graded 0,2 , or 4 ) was adequately described in 4 studies, not adequately described in 14 studies, and not described in 0 studies.

\section{Current Rehabilitation Protocols}

Rehabilitation protocols were variably and poorly reported (Table 1). Allowance of immediate weight bearing as tolerated 
TABLE 1 | Rehabilitation protocols used in all analyzed studies

\begin{tabular}{|c|c|c|c|c|c|}
\hline Study & Weight-bearing status & WBAT permitted & CPM use & Brace use & $\begin{array}{c}\text { Anti-rotational } \\
\text { boots }\end{array}$ \\
\hline McDonald et al. (12) & $\begin{array}{l}\text { Flat-foot WB (max } \\
20 \text { lbs) } \times 8 \text { weeks }(\mathrm{Mfx}) \\
\text { Flat-foot WB (max } \\
20 \text { lbs) } \times 2 \text { weeks (no Mfx })\end{array}$ & $\begin{array}{l}8 \text { weeks (Mfx) } \\
2 \text { weeks (no Mfx) }\end{array}$ & $\begin{array}{l}6-8 \mathrm{~h} / \text { day } \times \\
8 \text { weeks }(\mathrm{Mfx}) \\
6-8 \mathrm{~h} / \text { day } \times \\
3 \text { weeks (no Mfx) }\end{array}$ & $\begin{array}{l}\text { Prevent hip extension and } \\
\text { external rotation; } 10-21 \text { days; } \\
\text { while ambulating }\end{array}$ & 2 weeks \\
\hline Krych et al. (3) & Flat-foot PWB & 2 weeks & - & - & - \\
\hline McCormick et al. (13) & Flat-foot WB & Immediately post-operatively & - & - & - \\
\hline Kalore and Jiranek (14) & $50 \% \mathrm{WB} \times 1$ week & 1 week & - & - & - \\
\hline Philippon et al. (15) & PWB $\times 2-3$ weeks & 2-3 weeks & - & - & 3 weeks \\
\hline Malviya et al. (4) & PWB $\times 4$ weeks & 4 weeks & - & - & - \\
\hline Stafford et al. (16) & TTWB $\times 4$ weeks & 4 weeks & - & - & - \\
\hline Byrd and Jones (17) & $\begin{array}{l}\text { WBAT (unless Mfx, then } \\
\text { protected } \times 2 \text { months) }\end{array}$ & $\begin{array}{l}\text { Immediately post-operatively } \\
\text { (no Mfx) }\end{array}$ & - & - & - \\
\hline Marchie et al. (18) & WBAT & Immediately post-operatively & - & No & No \\
\hline Nho et al. (19) & $\begin{array}{l}20 \mathrm{lbs} \text { foot-flat } \\
\text { WB } \times 2-3 \text { weeks }\end{array}$ & 3 weeks & 4 h/day & Yes $\times 6$ weeks & - \\
\hline Haviv and O'Donnell (20) & WBAT & Immediately post-operatively & - & - & - \\
\hline Horisberger et al. (21) & $\begin{array}{l}\text { WBAT (unless Mfx: } 15-20 \mathrm{~kg} \\
\text { WB for } 4-6 \text { weeks) }\end{array}$ & $\begin{array}{l}\text { Immediately post-operatively } \\
\text { (no Mfx) }\end{array}$ & - & - & - \\
\hline Streich et al. (22) & Toe-touch WB $10 \mathrm{~kg} \times$ weeks & 2 weeks & - & - & - \\
\hline Philippon et al. (23) & 20 lbs WB (for 6-8 weeks if Mfx) & $\mathrm{Nr}$ & $\begin{array}{l}8-12 \mathrm{~h} / \text { day } \times \\
4 \text { weeks }\end{array}$ & 10 days & 10 days \\
\hline Kim et al. (24) & WBAT & Immediately post-operatively & - & - & - \\
\hline Fox (25) & WBAT & Immediately post-operatively & - & - & - \\
\hline O'Leary et al. (26) & WBAT & Immediately post-operatively & - & - & - \\
\hline Farjo et al. (27) & WBAT & Immediately post-operatively & - & - & - \\
\hline
\end{tabular}

following surgery was reported in seven studies when treatment was labral debridement, synovial chondromatosis loose body removal, osteoarthritis debridement, septic arthritis debridement, and trochanteric bursectomy. When labral repair, femoral osteochondroplasty, and pincer acetabuloplasty were performed, a partial weight-bearing protocol was initiated. Three studies described partial weight bearing as "foot-flat," while two described it as "toetouch" or "touchdown." Performance of microfracture warranted partial weight bearing for 4-8 weeks in four studies. Use of CPM was reported in only three studies, with between 4 and $12 \mathrm{~h} /$ day use for between 4 and 8 weeks. Brace/orthosis use was reported in only four studies: one study denied the use of a brace, two reported only the duration of time used (10 days, 6 weeks), and the other one did report the duration (10-21 days) and motion restrictions (prevent hip extension and external rotation) and situation (while ambulating). Anti-rotational boot use was reported in only four studies: one study denied their use, and the other three only reported the duration of time used (10 days, 2 and 3 weeks). Only five studies reported the permission and progression to return-tosport protocols (Table 2). Initiation of low-impact sports began at 6 weeks at the earliest and high-impact sports between 3 and 6 months.

Four studies (Table 3) recommended specific phase-based rehabilitation protocols following hip arthroscopy (28-31). All four studies described four phases that generally reported formal timeline-based (Table 3) and criteria-based (Table 4) protocols
TABLE 2 | Description of permission to RTS in all studies analyzed.

\begin{tabular}{ll}
\hline Study & Permit RTS \\
\hline McDonald et al. (12) & Impact sports at 3-6 months \\
$\begin{array}{l}\text { Krych et al. (3) } \\
\text { McCormick et al. (13) }\end{array}$ & $\begin{array}{l}\text { Impact loading exercises and deep squatting } \\
\text { allowed at 4 months }\end{array}$ \\
Kalore and Jiranek (14) & - \\
Philippon et al. (15) & - \\
Malviya et al. (4) & -
\end{tabular}

Stafford et al. (16)

Resume pre-operative activity levels at 3 months

Byrd and Jones (17)

Marchie et al. (18)

Nho et al. (19)

Haviv and O'Donnell (20)

Horisberger et al. (21)

Low-impact RTS at 6 weeks; high-impact sports

Streich et al. (22)

Philippon et al. (23)

Kim et al. (24)

Fox (25)

O'Leary et al. (26)

Farjo et al. (27) at 3 months

Impact loading allowed at 3 months
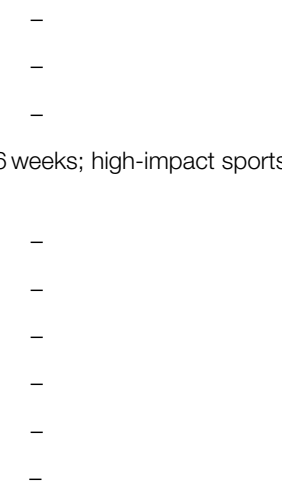


\begin{tabular}{lll}
\hline & Phase I & Phase II \\
\hline Edelstein & 0-6 weeks post-op & 4-12 weeks post-op \\
et al. (29) & 20\% foot-flat WB $\times 2$ weeks & Re-education of psoas, using eccentric exercises \\
& If microfracture or gluteus medius repair, foot-flat WB & Re-education of transversus abdominis firing \\
& 6 weeks & Gluteal and pelvic/hip strengthening \\
& No ROM restrictions unless capsular repair or iliopsoas & \\
& release & \\
& CPM $\times 3$ weeks, brace $\times 10$ days & \\
& Manual skills, soft-tissue mobilization & \\
\hline
\end{tabular}

\section{Wahoff and Foot-flat WB $\times 3$ weeks (no Mfx) \\ Ryan (30)}

Foot-flat WB $\times 6-8$ weeks (Mfx)

Brace limiting external rotation, extension $\times 3$ weeks

CPM $30-70^{\circ}$ in $10^{\circ}$ abduction, $4-6 \mathrm{~h} /$ day $\times 2$ weeks (Mfx 6-8 weeks)

Stationary bike 20 minutes $1-2 \times /$ day $\times 6$ weeks

Circumduction $2 \times /$ day $\times 2$ weeks; $1 \times /$ day $\times 10$ weeks

Prone lying $\times 2 \mathrm{~h} /$ day

Isometrics quads, gluteus maximus, transverse abdominis Deep soft-tissue massage
Voight et al. Variable WB status - if biological healing required, foot-flat

(28)

\section{WB 8-10 weeks; otherwise WBAT within 1 week}

Restore passive ROM, especially internal rotation and

flexion - prevent adhesions

Stretching only to tolerance, not beyond

Stationary bike without resistance

Isometrics of gluts, quads, adductor, abductor, hamstrings abdominals

Aquatic program

Garrison Weeks 0-4

et al. (31) $\quad 50 \%$ WB for $7-10$ days (unless labral repair - toe-touch WB $\times$ 3-6 weeks)

Flexion limited to $90^{\circ}$ for 2 weeks (no limit extension, rotation, or abduction) for labral debridement

Flexion limited to $90^{\circ}$ for 2 weeks, extension to $10^{\circ}$ for 2 weeks, rotation gentle for 2 weeks, abduction $25^{\circ} 2$ weeks Prone lying $1-2 \mathrm{~h} /$ day

Stationary bike without resistance

Isometrics abductors, adductors, extensors, transverse abdominals

\section{Phase III}

\section{Phase IV}

8-20 weeks post-op

Re-build strength, endurance

12-28 weeks post-op

Core control during all activities

sive power

High, low velocity strength

ncrease volume, intensity of aerobic Sport-specific speed

activities

Proprioception on varying surfaces, Repetition work

with perturbations

Plyometrics (able to squat 150\% BW) Incorporation of rest time

Wean off crutches (depending on WB status - $\pm \mathrm{Mfx}$ )

Continue circumduction, prone

soft-tissue mobilization

Gluteal activation and core and pelvis Sport-specific training

Continue circumduction, prone lying

stabilization

Double-leg strengthening advancement Power, plyometric, performance to single-leg strengthening

Sport progressions to functional

activities

Restored cardiovascular fitness

Advanced power, plyometrics,

performance, conditioning

\section{Pilates recommended vs, yoga}

Reassure mental and physical rehabilitation

Add resistance to cycling at week 6

Begins at week 4

\section{Pain-free full ROM}

Continue strengthening and stabilization

\section{Add WB and resistance exercises}

Address muscle imbalances: tight hip flexors and erecto spinae, weak gluteals and abs (forward pelvic tilt and lumbar lordosis increase)

Core stabilization and strengthening

\section{Weeks 5-7}

Emphasis shifts from motion to strength

Continue manual therapy

\section{Aquatic therapy}

Kneeling hip flexor stretch once tolerated

Passive ROM should become more aggressive, especially rotation

Hip and core and pelvis strengthening

Add resistance to bike

Build cardiovascular endurance

\section{Proprioceptive re-training}

Dynamic stabilization exercises, encouraging co-contractions Begin advanced strengthening in poo before land

Progress exercises

Slow to fast

\section{Simple to complex}

Stable to unstable

Low to high force

\section{Weeks 8-12}

Integrated functional strengthening

Manual therapy as needed

If full ROM not achieved by week 10 , terminal stretches should be initiated

Multi-planar muscle strengthening

Core strengthening

Plyometrics in water

Running at end of phase

Agility drills
Weeks $12+$

\section{Return to sports}

Individualized based on hip pathology and surgery performed

\section{Safe, effective return to sports}

Careful, frequent re-assessmen to prevent loss of mobility as strengthening continues to advance 
TABLE 4 | Criteria-based progression from phase to phase in post-operative rehabilitation

\begin{tabular}{|c|c|c|c|c|}
\hline & Phase I-II & Phase II-III & Phase III-IV & Phase IV to unrestricted sports \\
\hline $\begin{array}{l}\text { Edelstein } \\
\text { et al. (29) }\end{array}$ & $\begin{array}{l}\text { Normalized gait without assistance } \\
\text { No Trendelenberg } \\
80 \% \text { full ROM } \\
\text { Core stabilization }\end{array}$ & $\begin{array}{l}\text { Normal ADL's without pain } \\
\text { Full ROM } \\
\text { Core stability Sahrmann } 2 \times 30 \text { s } \\
5 / 5 \text { manual muscle strength } \\
\text { Good control in single-leg squat }\end{array}$ & $\begin{array}{l}\text { Recreationally asymptomatic } \\
\text { Maintenance of core control } \\
\text { "10 rep triple" }\end{array}$ & $\begin{array}{l}\text { Pain-free competitive state } \\
\text { Micromanagement of return to sport to } \\
\text { consistently and painlessly perform } \\
\text { motion responsible for initial injury }\end{array}$ \\
\hline \multirow{2}{*}{$\begin{array}{l}\text { Wahoff } \\
\text { and Ryan } \\
\text { (30) }\end{array}$} & Minimal pain with all Phase I & Pain-free normal gait & $\begin{array}{l}\text { Passing of a sports test, allowing } \\
\text { return to practice without limitations }\end{array}$ & Physician clearance \\
\hline & $\begin{array}{l}\text { Minimal "pinching" before } 100^{\circ} \\
\text { flexion } \\
\text { Tolerated full WB }\end{array}$ & $\begin{array}{l}\text { Full ROM } \\
\text { Core, pelvic stability } \\
\text { Balance, proprioception }\end{array}$ & $\begin{array}{l}\text { Perform all Phase III exercises pain } \\
\text { free and with correct form }\end{array}$ & $\begin{array}{l}\text { Full return to practice without } \\
\text { restrictions }\end{array}$ \\
\hline $\begin{array}{l}\text { Voight } \\
\text { et al. (28) }\end{array}$ & $\begin{array}{l}\text { Close to full ROM } \\
\text { Normalized gait without crutches } \\
\text { Minimal to no pain }\end{array}$ & $\begin{array}{l}\text { Pelvic tilt test, pelvic rotation test, } \\
\text { torso rotation test, bridge with } \\
\text { leg extension test }\end{array}$ & $\begin{array}{l}\text { Proprioceptive and neuromuscular } \\
\text { control }\end{array}$ & $\begin{array}{l}\text { Depends on hip pathology treated and } \\
\text { specific demands of sport played }\end{array}$ \\
\hline \multirow[t]{3}{*}{$\begin{array}{l}\text { Garrison } \\
\text { et al. (31) }\end{array}$} & $\mathrm{ROM} \geq 75 \%$ contralateral side & $\begin{array}{l}\text { Normal gait without } \\
\text { Trendelenberg sign }\end{array}$ & Symmetric motion & $\begin{array}{l}\text { Completion of return-to-play test using } \\
\text { sportcord test }\end{array}$ \\
\hline & $\begin{array}{l}\text { Ability to do side-lying straight-leg } \\
\text { raise }\end{array}$ & Symmetric passive ROM & $\begin{array}{l}\text { Symmetric flexibility of psoas, } \\
\text { piriformis }\end{array}$ & $\begin{array}{l}\text { Dynamic functional activities with } \\
\text { resistance from sportcord: single-leg }\end{array}$ \\
\hline & & No pain & $\begin{array}{l}\text { No Trendelenberg with higher level } \\
\text { functional strengthening }\end{array}$ & $\begin{array}{l}\text { squat } \times 3 \text { min, lateral bounding } \times 80 \mathrm{~s} \text {, } \\
\text { forward/backward jogging } \times 2 \mathrm{~min}\end{array}$ \\
\hline
\end{tabular}

TABLE 5 | Precautions recommended at each phase in post-operative rehabilitation.

\begin{tabular}{|c|c|c|c|c|}
\hline & Phase I & Phase II & Phase III & Phase IV \\
\hline $\begin{array}{l}\text { Edelstein } \\
\text { et al. (29) }\end{array}$ & $\begin{array}{l}\text { Not lifting leg on its own } \\
\text { Not crossing legs } \\
\text { Not pushing ROM to point of pain }\end{array}$ & $\begin{array}{l}\text { Avoid hip flexor tendonitis } \\
\text { (iliopsoas, TFL, sartorius, } \\
\text { rectus femoris) }\end{array}$ & $\begin{array}{l}\text { Avoid sacrificing quality for } \\
\text { quantity during strengthening }\end{array}$ & $\begin{array}{l}\text { Avoid breakdown to acute } \\
\text { inflammatory response }\end{array}$ \\
\hline $\begin{array}{l}\text { Wahoff } \\
\text { and Ryan } \\
\text { (30) }\end{array}$ & $\begin{array}{l}\text { No hip extension past neutral } \times 3 \text { weeks } \\
\text { No external rotation } \times 3 \text { weeks } \\
\text { No flexion beyond } 120^{\circ} \\
\text { No abduction beyond } 45^{\circ}\end{array}$ & $\begin{array}{l}\text { Avoid treadmill (shear stress) } \\
\text { Avoid hip flexor and adductor } \\
\text { inflammation } \\
\text { Avoid ballistic stretching }\end{array}$ & $\begin{array}{l}\text { Avoid treadmill } \\
\text { Avoid hip flexor and adductor } \\
\text { inflammation } \\
\text { Avoid ballistic stretching and } \\
\text { high-velocity activities }\end{array}$ & None \\
\hline $\begin{array}{l}\text { Voight } \\
\text { et al. (28) }\end{array}$ & $\begin{array}{l}\text { No recumbent bike } \\
\text { No aerodynamic bike riding position }\end{array}$ & $\begin{array}{l}\text { Avoid arthrokinetic inhibition } \\
\text { Avoid synergistic dominance } \\
\text { Avoid reciprocal inhibition }\end{array}$ & $\begin{array}{l}\text { Depends on tolerance to } \\
\text { advancement of activities }\end{array}$ & $\begin{array}{l}\text { Avoid compressive forces generated } \\
\text { by sports, depending on hip pathology } \\
\text { and surgical treatment }\end{array}$ \\
\hline $\begin{array}{l}\text { Garrison } \\
\text { et al. (31) }\end{array}$ & $\begin{array}{l}\text { Avoid tight hip flexors and erector spinae } \\
\text { Avoid inhibited gluts and abs } \\
\text { Avoid hip flexion straight-leg raises to } \\
\text { avoid hip flexor tendonitis }\end{array}$ & Avoid pain & $\begin{array}{l}\text { Avoid any loss of motion } \\
\text { Avoid loss of core strength }\end{array}$ & $\begin{array}{l}\text { Avoid loss of flexibility as strength } \\
\text { continues to increase }\end{array}$ \\
\hline
\end{tabular}

with precautions (Table 5) advised during each phase. Phase I was a period of protection, between 0 and 6 weeks following surgery, with limited weight bearing, restoration of early motion, limited core abdominopelvic, and hip isometric strengthening, with avoidance of excessive hip extension (beyond neutral), external rotation, deep flexion, and iliopsoas tendonitis. Phase II was a period of advancement to pain-free normal weight bearing and gait and motion, between 4 and 12 weeks postoperatively. Recommendations were for continued strengthening of core and hip muscles, while still avoiding hip flexor tendonitis. Phase III ranged between 8 and 20 weeks after surgery, with focus on endurance, in addition to strength, and progression to sport-specific training. Advancement to Phase IV generally required pain-free full motion, strength, without any subjective or objective deficits during training. Phase IV began at a minimum of 12 weeks following surgery, with progression to safe and unrestricted return to normal activities and sports as well as avoidance of any regression to pain, stiffness, or weakness. All four studies also described a permission to return to running and unrestricted sports protocols (Table 6). One study reported an explicit requirement of passage of a return-to-sport test to permit running and a different study reported an explicit requirement of passage of a test to permit unrestricted return to sports.

\section{Clinical Outcomes}

Clinical outcomes were variably and poorly reported (Table 7). Significant improvements were demonstrated for multiple 
TABLE 6 | Criteria-based permission to return to running and return to sports described in each study.

\begin{tabular}{|c|c|c|}
\hline & Permission to run & Unrestricted sports \\
\hline Edelstein et al. (29) & $\begin{array}{l}\text { "10-rep triple": } 10 \text { front step-downs and } 10 \text { single-leg } \\
\text { squats without kinetic collapse, } 10 \text { side-lying leg raises } \\
\text { against resistance with at least } 4 / 5 \text { manual muscle strength }\end{array}$ & $\begin{array}{l}\text { Consistent and painless repetitions of the movement responsible for the } \\
\text { mechanism of injury }\end{array}$ \\
\hline Wahoff and Ryan (30) & $\begin{array}{l}\text { Pain-free, progressive, predictable } \\
\text { Initiate pool running several weeks prior to land in runners }\end{array}$ & Physician clearance after return to unrestricted practice \\
\hline Voight et al. (28) & Not reported & Depends on hip pathology and surgical treatment performed \\
\hline Garrison et al. (31) & $\begin{array}{l}\text { Pool running at } 2-3 \text { weeks } \\
\text { Once good eccentric control, muscular endurance, ability to } \\
\text { generate power }\end{array}$ & $\begin{array}{l}\text { Completion of return-to-play test using sportcord test }- \text { Dynamic functional } \\
\text { activities with resistance from sportcord: single-leg squat } \times 3 \text { min, lateral } \\
\text { bounding } \times 80 \mathrm{~s} \text {, forward/backward jogging } \times 2 \text { min }\end{array}$ \\
\hline
\end{tabular}

TABLE 7 | Salient outcomes in all studies analyzed.

\begin{tabular}{|c|c|c|c|c|c|}
\hline Study & $\begin{array}{l}\text { Level of } \\
\text { evidence }\end{array}$ & $\begin{array}{c}\text { Subject } \\
\text { population }\end{array}$ & $\begin{array}{l}\text { Study } \\
\text { design }\end{array}$ & Intervention & Primary outcome \\
\hline $\begin{array}{l}\text { McDonald } \\
\text { et al. (12) }\end{array}$ & 3 & Elite athletes & $\begin{array}{l}\text { Case- } \\
\text { control }\end{array}$ & $\begin{array}{l}\text { Microfracture (case) vs. no } \\
\text { microfracture (control) }\end{array}$ & $\begin{array}{l}\text { - Return to sport: } 77 \% \text { in microfracture vs. } 84 \% \text { in } \\
\text { non-microfracture }(p>0.05)\end{array}$ \\
\hline $\begin{array}{l}\text { Krych et al. } \\
\text { (3) }\end{array}$ & 1 & Females & $\mathrm{RCT}$ & Labral repair vs. debridement & $\begin{array}{l}\text { - Better HOS (ADL, sport) in repair group ( } p<0.05 \text { for both) } \\
\text { - Better subjective outcome in repair group ( } p<0.05)\end{array}$ \\
\hline $\begin{array}{l}\text { McCormick } \\
\text { et al. (13) }\end{array}$ & 3 & $\begin{array}{l}\text { Patients with } \\
\text { labral tears }\end{array}$ & $\begin{array}{l}\text { Case- } \\
\text { control }\end{array}$ & Labral repair vs. debridement & $\begin{array}{l}\text { - Presence of OA at arthroscopy predictive of worse outcomes } \\
\text { - Age }>40 \text { years predictive of worse outcomes }\end{array}$ \\
\hline $\begin{array}{l}\text { Kalore and } \\
\text { Jiranek (14) }\end{array}$ & 4 & $\begin{array}{l}\text { Patients with } \\
\text { labral tears }\end{array}$ & $\begin{array}{l}\text { Case } \\
\text { series }\end{array}$ & Labral repair vs. debridement & $\begin{array}{l}\text { - Higher }(p<0.05) \text { re-operation rate in } \\
\text { o Borderline vs. adequate acetabular coverage } \\
\circ \text { Labral debridement vs. repair }\end{array}$ \\
\hline $\begin{array}{l}\text { Philippon } \\
\text { et al. (15) }\end{array}$ & 4 & $\begin{array}{l}\text { FAl, } 11-16 \text { years } \\
\text { of age }\end{array}$ & $\begin{array}{l}\text { Case } \\
\text { series }\end{array}$ & FAl and labral treatment & $\begin{array}{l}\text { - Significant }(p<0.05) \text { improvement in mHHS (57-91 at 3years) } \\
\text { - } 8 / 60 \text { (13\%; all girls) needed repeat arthroscopy (adhesions) }\end{array}$ \\
\hline $\begin{array}{l}\text { Malviya et al. } \\
\text { (4) }\end{array}$ & 4 & $\begin{array}{l}\text { FAl, } 14-75 \text { years } \\
\text { of age }\end{array}$ & $\begin{array}{l}\text { Case } \\
\text { series }\end{array}$ & FAl and labral treatment & $\begin{array}{l}\text { - Significant }(p<0.05) \text { improvement in } \mathrm{QoL} \\
\text { - } 74 \% \text { of patients happy with results }\end{array}$ \\
\hline $\begin{array}{l}\text { Stafford et al. } \\
\text { (16) }\end{array}$ & 4 & $\begin{array}{l}\text { FAl, chondral } \\
\text { defect } \\
\text { acetabulum }\end{array}$ & $\begin{array}{l}\text { Case } \\
\text { series }\end{array}$ & $\begin{array}{l}\text { Microfracture with repair of } \\
\text { delaminated cartilage using } \\
\text { fibrin adhesive }\end{array}$ & - Significant $(p<0.001)$ improvement in $\mathrm{mHHS}$ at 2 years \\
\hline $\begin{array}{l}\text { Byrd and } \\
\text { Jones (17) }\end{array}$ & 4 & FAl & $\begin{array}{l}\text { Case } \\
\text { series }\end{array}$ & FAI and labral treatment & - Significant $(p<0.001)$ improvement in $\mathrm{mHHS}$ at 2 years \\
\hline $\begin{array}{l}\text { Marchie } \\
\text { et al. (18) }\end{array}$ & 4 & $\begin{array}{l}\text { Synovial } \\
\text { chondromatosis }\end{array}$ & $\begin{array}{l}\text { Case } \\
\text { series }\end{array}$ & Loose body removal & $\begin{array}{l}\text { - } 48 \% \text { good/excellent outcomes at } 5.3 \text { years } \\
\text { - } 17 \% \text { underwent total hip arthroplasty at mean } 4.3 \text { years }\end{array}$ \\
\hline $\begin{array}{l}\text { Nho et al. } \\
\text { (19) }\end{array}$ & 4 & $\begin{array}{l}\text { High-level } \\
\text { athletes, FAl }\end{array}$ & $\begin{array}{l}\text { Case } \\
\text { series }\end{array}$ & FAl and labral treatment & $\begin{array}{l}\text { - Significant improvements in } \mathrm{mHHS} \text { and HOS at } 2 \text { years } \\
\text { - } 79 \% \text { return to sports at mean } 9.4 \text { months }\end{array}$ \\
\hline $\begin{array}{l}\text { Haviv and } \\
\text { O'Donnell } \\
\text { (20) }\end{array}$ & 4 & Osteoarthritis & $\begin{array}{l}\text { Case } \\
\text { series }\end{array}$ & FAl and labral treatment & $\begin{array}{l}\text { - } 16 \% \text { of patients eventually underwent total hip arthroplasty } \\
\text { - Age }<55 \text { years and mild osteoarthritis predictive of longer time to } \\
\text { arthroplasty }\end{array}$ \\
\hline $\begin{array}{l}\text { Horisberger } \\
\text { et al. }(21)\end{array}$ & 4 & Osteoarthritis & $\begin{array}{l}\text { Case } \\
\text { series }\end{array}$ & FAl and labral treatment & $\begin{array}{l}\text { - } 40 \% \text { of patients eventually underwent total hip arthroplasty } \\
\text { - Mean index time to arthroplasty was } 1.4 \text { years (range } 0.4-2.2 \text { ) }\end{array}$ \\
\hline $\begin{array}{l}\text { Streich et al. } \\
(22)\end{array}$ & 4 & $\begin{array}{l}\text { Labral tears, no } \\
\text { FAl }\end{array}$ & $\begin{array}{l}\text { Case } \\
\text { series }\end{array}$ & Labral treatment & $\begin{array}{l}\text { - Significant improvements in Larson hip score and mHHS } \\
\text { - Presence of acetabular chondral defect worse prognosis }\end{array}$ \\
\hline $\begin{array}{l}\text { Philippon } \\
\text { et al. (23) }\end{array}$ & 4 & $\begin{array}{l}\text { FAl, 38-44 years } \\
\text { of age }\end{array}$ & $\begin{array}{l}\text { Case } \\
\text { series }\end{array}$ & FAl and labral treatment & $\begin{array}{l}\text { - Significant improvements in mHHS at } 2 \text { years } \\
\text { - } 11 \% \text { of patients underwent total hip arthroplasty at mean } \\
16 \text { months }\end{array}$ \\
\hline $\begin{array}{l}\text { Kim et al. } \\
(24)\end{array}$ & 4 & Septic arthritis & $\begin{array}{l}\text { Case } \\
\text { series }\end{array}$ & $\begin{array}{l}\text { Arthroscopic debridement } \\
\text { and drainage }\end{array}$ & $\begin{array}{l}\text { - Excellent results obtained at } 4.9 \text { years } \\
\text { - No complications, no re-operations }\end{array}$ \\
\hline Fox (25) & 4 & $\begin{array}{l}\text { Trochanteric } \\
\text { bursitis }\end{array}$ & $\begin{array}{l}\text { Case } \\
\text { series }\end{array}$ & Trochanteric bursectomy & $\begin{array}{l}\text { - } 85 \% \text { excellent/good results at } 5 \text { years; } 96 \% \text { satisfaction } \\
\text { - Only } 2 \text { recurrences of pain }\end{array}$ \\
\hline $\begin{array}{l}\text { O'Leary et al. } \\
\text { (26) }\end{array}$ & 4 & Various & $\begin{array}{l}\text { Case } \\
\text { series }\end{array}$ & $\begin{array}{l}\text { Various arthroscopic } \\
\text { techniques }\end{array}$ & $\begin{array}{l}\text { - } 60 \% \text { significant improvements at } 2.5 \text { years } \\
\text { - OA and AVN had significantly worse outcomes (vs. labral tears) } \\
\text { - } 21 \% \text { underwent total hip arthroplasty at mean } 8.4 \text { months }\end{array}$ \\
\hline $\begin{array}{l}\text { Farjo et al. } \\
(27)\end{array}$ & 4 & Labral tear & $\begin{array}{l}\text { Case } \\
\text { series }\end{array}$ & Labral debridement & $\begin{array}{l}\text { - } 46 \% \text { good, } 54 \% \text { poor results } \\
\text { - } 29 \% \text { underwent total hip arthroplasty at mean } 23 \text { months }\end{array}$ \\
\hline
\end{tabular}


diagnoses treated with various surgical techniques utilizing NAHS, HOS, HOOS, and mHHS. However, given the heterogeneity between subjects and surgeries performed, no comparison could be made between any group of subjects based on the rehabilitation protocol following surgery.

\section{Discussion}

The purposes of this systematic review were to determine the current rehabilitation protocols utilized following hip arthroscopy in the current literature, if clinical outcomes are significantly different based on different post-operative rehabilitation protocols, and to propose the best-available evidence-based rehabilitation program following hip arthroscopy. The authors hypothesized that post-operative rehabilitation protocols are infrequently and poorly reported with significant heterogeneity. The authors also hypothesized that there is little to no evidence that supports or refutes specific post-operative rehabilitation protocols and that current protocols are based on theory and biomechanical, rather than clinical, investigations. The study hypotheses were confirmed, thus strengthening the previous assertion by Cheatham et al. that there is a paucity of evidence surrounding post-operative rehabilitation protocols following hip arthroscopy (32).

Rehabilitation following hip arthroscopy is an integral part of a successful outcome in treatment of various intra- and extraarticular hip pathologies. The current medical climate mandates assimilation of evidence-based medicine and patient-reported outcomes into everyday clinical practice. This includes assessment of basic science and clinical outcomes literature and incorporation of this evidence into discussions with patients. This mandates that the rehabilitation literature following hip arthroscopy significantly improve. The authors selected clinical follow-up studies with minimum 2-year follow-up to accurately identify current rehabilitation protocols. Although 18 studies were identified for inclusion and analyzed, nearly twice as many studies $(n=34)$ would have also been included (Figure 1), but those studies did not report a single word about rehabilitation in the entirety of the study. Even within the 18 studies included for final analysis, evaluation of the quality of their reporting was poor (via MCMS) and significant heterogeneity was demonstrated. Little recognition of the importance of rehabilitation was exhibited in the current literature. This does not necessarily mean that the quality of rehabilitation or the conduct of the trial is poor, only that the quality of reporting is poor.

Given the inability to extract evidence-based guidelines from clinical outcome studies of hip arthroscopy rehabilitation in this systematic review, the authors utilized narrative review articles (Tables 3-6) to summarize and report the best-available evidence on the topic.

\section{Principles of Rehabilitation}

Rehabilitation following hip arthroscopy should be individualized and evaluation based rather than time based. Circumduction is key in enhancing early motion and preventing intra- and extraarticular adhesions. Weight bearing and motion progression is based upon the specific surgical techniques performed. Thus, a "cookbook" rehabilitation program after arthroscopic surgery of the hip is not recommended. Nevertheless, when protection or biological healing is required (labral repair, capsular repair or plication, femoral osteochondroplasty), rehabilitation should progress more slowly vs. procedures in which no protection or healing is needed (labral debridement, loose body removal, ligamentum teres debridement, synovectomy). Avoidance of hip flexor tendonitis is recommended throughout rehabilitation [not only primary hip flexors (iliopsoas) but also secondary flexors (rectus femoris, sartorius, tensor fascia lata)]. Given that the iliopsoas is largely inhibited early after surgery, the activation and overactivation of secondary flexors may occur, thus relegating them to potential inflammatory overuse.

Patients undergoing hip arthroscopy are young (mean age 35 years in this review) and active. As such, rehabilitation protocol efficacy should be assessed using patient-reported outcome instruments that are appropriate for use in this patient population. HOS, the International Hip Outcome Tool (iHOT33/iHOT-12), and the Copenhagen hip and groin outcome score (HAGOS) have been recommended to guide therapy progression (33). Wahoff et al. described a comprehensive, criteria-driven algorithm for safe integration and return to sport rehabilitation following hip arthroscopy. Emphasis is placed on various criteria to advance through the six phases including healing restraints, patient-reported outcomes, range of motion, and other sportspecific tasks. As a part of the minimum criteria for advancement, the HOS was chosen as it contains both ADL and sports subscales. These separate scales make it appropriate for use in both early rehabilitation and late as it is responsive during higher levels of physical ability (34).

Return to sport is a very relevant component of the surgical outcome. Too early return may lead to recurrence of pain. Progression through phases of rehabilitation necessitates meeting specific goals and milestones as described above. Passing these thresholds improves the likelihood of safe return to sport. Return-to-sport tests are gaining acceptance in return to play following ACL reconstruction $(35,36)$. The same standards should be applied to patients undergoing hip arthroscopy, as the subject demographics, rehabilitation timelines, and sport goals are similar.

\section{Limitations}

The limitations of any systematic review are dependent upon the included studies, which it analyzes. Selection bias in this review was minimized by the inclusive nature of study selection. However, bias is also recognized by exclusion of studies with $<2$ years follow-up. Performance bias was also minimized by the inclusive nature of study selection, allowing all subject diagnoses and surgical treatments available to be included. It is recognized, however, that no study reported subject compliance with rehabilitation, including weight-bearing status, motion restrictions, CPM use, brace or boot use, or return to sports. Heterogeneity in definitions of rehabilitation phases, protocols, goals, precautions, and return to sport variables introduces detection bias. Study design bias is present in the retrospective nature of 17 out of 18 (94\%) included studies. Publication bias is present in that the authors excluded medical conference abstracts, non-English language studies, and non-published English language studies. 


\section{Conclusion}

The current literature of hip arthroscopy rehabilitation lacks high-quality evidence to support a specific protocol. Heterogeneity in study, subject, and surgical demographics

\section{References}

1. Ganz R, Parvizi J, Beck M, Leunig M, Notzli H, Siebenrock KA. Femoroacetabular impingement: a cause for osteoarthritis of the hip. Clin Orthop Relat Res (2003) 417:112-20.

2. Larson CM, Giveans MR, Stone RM. Arthroscopic debridement versus refixation of the acetabular labrum associated with femoroacetabular impingement: mean 3.5-year follow-up. Am J Sports Med (2012) 40(5):1015-21. doi:10.1177/ 0363546511434578

3. Krych AJ, Thompson M, Knutson Z, Scoon J, Coleman SH. Arthroscopic labral repair versus selective labral debridement in female patients with femoroacetabular impingement: a prospective randomized study. Arthroscopy (2013) 29(1):46-53. doi:10.1016/j.arthro.2012.07.011

4. Malviya A, Stafford GH, Villar RN. Impact of arthroscopy of the hip for femoroacetabular impingement on quality of life at a mean follow-up of 3.2 years. J Bone Joint Surg Br (2012) 94(4):466-70. doi:10.1302/0301-620X.94B4. 28023

5. Tanzer M, Noiseux N. Osseous abnormalities and early osteoarthritis: the role of hip impingement. Clin Orthop Relat Res (2004) 429:170-7. doi:10.1097/01. blo.0000150119.49983.ef

6. Bedi A, Chen N, Robertson W, Kelly BT. The management of labral tears and femoroacetabular impingement of the hip in the young, active patient. Arthroscopy (2008) 24(10):1135-45. doi:10.1016/j.arthro.2008.06.001

7. Montgomery S, Ngo SS, Hobson T, Nguyen S, Alluri R, Wang JC, et al. Trends and demographics in hip arthroscopy in the United States. Arthroscopy (2013) 29(4):661-5. doi:10.1016/j.arthro.2012.11.005

8. Moher D, Liberati A, Tetzlaff J, Altman DG. Preferred reporting items for systematic reviews and meta-analyses: the PRISMA statement. J Clin Epidemiol (2009) 62(10):1006-12. doi:10.1016/j.jclinepi.2009.06.005

9. York CfRaD-TUo. PROSPERO - International Prospective Register of Systematic Reviews (2012). Available from: http://www.crd.york.ac.uk/PROSPERO/ (accessed January 29, 2103).

10. Oxford C-Uo. OCEBM 2011 Levels of Evidence System. Oxford Centre for Evidence Based Medicine (2012). Available from: http://www.cebm.net/index. aspx? $=5653$ (accessed December 20, 2012).

11. Coleman B, Khan K, Maffulli N, Cook J, Wark J. Studies of surgical outcome after patellar tendinopathy: clinical significance of methodological deficiencies and guidelines for future studies. Scand J Med Sci Sports (2000) 10:2-11. doi:10. 1034/j.1600-0838.2000.010001002.x

12. McDonald JE, Herzog MM, Philippon MJ. Return to play after hip arthroscopy with microfracture in elite athletes. Arthroscopy (2013) 29(2):330-5. doi:10. 1016/j.arthro.2012.08.028

13. McCormick F, Nwachukwu BU, Alpaugh K, Martin SD. Predictors of hip arthroscopy outcomes for labral tears at minimum 2-year follow-up: the influence of age and arthritis. Arthroscopy (2012) 28(10):1359-64. doi:10.1016/j. arthro.2012.04.059

14. Kalore NV, Jiranek WA. Save the torn labrum in hips with borderline acetabular coverage. Clin Orthop Relat Res (2012) 470(12):3406-13. doi:10.1007/ s11999-012-2499-9

15. Philippon MJ, Ejnisman L, Ellis HB, Briggs KK. Outcomes 2 to 5 years following hip arthroscopy for femoroacetabular impingement in the patient aged 11 to 16 years. Arthroscopy (2012) 28(9):1255-61. doi:10.1016/j.arthro.2012.02.006

16. Stafford GH, Bunn JR, Villar RN. Arthroscopic repair of delaminated acetabular articular cartilage using fibrin adhesive. Results at one to three years. Hip Int (2011) 21(6):744-50. doi:10.5301/HIP.2011.8843

17. Byrd JW, Jones KS. Arthroscopic management of femoroacetabular impingement: minimum 2-year follow-up. Arthroscopy (2011) 27(10):1379-88. doi:10. 1016/j.arthro.2011.05.018

18. Marchie A, Panuncialman I, McCarthy JC. Efficacy of hip arthroscopy in the management of synovial chondromatosis. Am J Sports Med (2011) 39(Suppl):126S-31S. doi:10.1177/0363546511414014 precluded assimilation of protocols and/or outcomes to generate evidence-based guidelines. Strengths and limitations in the literature were identified. Future studies should recognize and report the essentials of rehabilitation following hip arthroscopy.

19. Nho SJ, Magennis EM, Singh CK, Kelly BT. Outcomes after the arthroscopic treatment of femoroacetabular impingement in a mixed group of high-level athletes. Am J Sports Med (2011) 39(Suppl):14S-9S. doi:10.1177/ 0363546511401900

20. Haviv B, O'Donnell J. The incidence of total hip arthroplasty after hip arthroscopy in osteoarthritic patients. Sports Med Arthrosc Rehabil Ther Technol (2010) 2:18. doi:10.1186/1758-2555-2-18

21. Horisberger M, Brunner A, Herzog RF. Arthroscopic treatment of femoral acetabular impingement in patients with preoperative generalized degenerative changes. Arthroscopy (2010) 26(5):623-9. doi:10.1016/j.arthro.2009.09.003

22. Streich NA, Gotterbarm T, Barie A, Schmitt H. Prognostic value of chondral defects on the outcome after arthroscopic treatment of acetabular labral tears. Knee Surg Sports Traumatol Arthrosc (2009) 17(10):1257-63. doi:10.1007/ s00167-009-0833-x

23. Philippon MJ, Briggs KK, Yen YM, Kuppersmith DA. Outcomes following hip arthroscopy for femoroacetabular impingement with associated chondrolabral dysfunction: minimum two-year follow-up. J Bone Joint Surg Br (2009) 91(1):16-23. doi:10.1302/0301-620X.91B1.21329

24. Kim SJ, Choi NH, Ko SH, Linton JA, Park HW. Arthroscopic treatment of septic arthritis of the hip. Clin Orthop Relat Res (2003) 407:211-4. doi:10.1097/ 00003086-200302000-00030

25. Fox JL. The role of arthroscopic bursectomy in the treatment of trochanteric bursitis. Arthroscopy (2002) 18(7):E34. doi:10.1053/jars.2002.35143

26. O'Leary JA, Berend K, Vail TP. The relationship between diagnosis and outcome in arthroscopy of the hip. Arthroscopy (2001) 17(2):181-8. doi:10.1053/jars. 2001.21481

27. Farjo LA, Glick JM, Sampson TG. Hip arthroscopy for acetabular labral tears. Arthroscopy (1999) 15(2):132-7. doi:10.1053/ar.1999.v15.015013

28. Voight ML, Robinson K, Gill L, Griffin K. Postoperative rehabilitation guidelines for hip arthroscopy in an active population. Sports Health (2010) 2(3):222-30. doi:10.1177/1941738110366383

29. Edelstein J, Ranawat A, Enseki KR, Yun RJ, Draovitch P. Post-operative guidelines following hip arthroscopy. Curr Rev Musculoskelet Med (2012) 5(1):15-23. doi:10.1007/s12178-011-9107-6

30. Wahoff M, Ryan M. Rehabilitation after hip femoroacetabular impingement arthroscopy. Clin Sports Med (2011) 30(2):463-82. doi:10.1016/j.csm. 2011.01.001

31. Garrison JC, Osler MT, Singleton SB. Rehabilitation after arthroscopy of an acetabular labral tear. N Am J Sports Phys Ther (2007) 2(4):241-50.

32. Cheatham SW, Enseki KR, Kolber MJ. Post-operative rehabilitation after hip arthroscopy: a search for the evidence. J Sport Rehabil (2014). doi:10.1123/jsr. 2014-0208

33. Enseki KR, Kohlrieser D. Rehabilitation following hip arthroscopy: an evolving process. Int J Sports Phys Ther (2014) 9(6):765-73.

34. Wahoff M, Dischavi S, Hodge J, Pharez JD. Rehabilitation after labral repair and femoroacetabular decompression: criteria-based progression through the return to sport phase. Int J Sports Phys Ther (2014) 9(6):813-26.

35. Barber-Westin SD, Noyes FR. Objective criteria for return to athletics after anterior cruciate ligament reconstruction and subsequent reinjury rates: a systematic review. Phys Sportsmed (2011) 39(3):100-10. doi:10.3810/psm.2011.09. 1926

36. Barber-Westin SD, Noyes FR. Factors used to determine return to unrestricted sports activities after anterior cruciate ligament reconstruction. Arthroscopy (2011) 27(12):1697-705. doi:10.1016/j.arthro.2011.09.009

Conflict of Interest Statement: Shane J. Nho is a paid consultant for Stryker, Pivot Medical, and Ossur; owns stock in Pivot Medical; and receives research support from Arthrex, Linvatec, Smith and Nephew, DJ Orthopaedics, Miomed, Athletico, Stryker, Pivot Medicine, and Allosource. Joshua David Harris is on editorial board for Arthroscopy: The Journal of Arthroscopic and Related Surgery; 
is a paid consultant for NIA Magellan; and receives royalties from SLACK, Inc. Charles Bush-Joseph is an unpaid consultant for The Foundry and is on the Medical Publications editorial/governing board for the American Journal of Sports Medicine. All other authors have no significant financial conflict of interest. However, the authors confirm that no financial conflict of interest influenced the topic of this manuscript.
Copyright (๑ 2015 Grzybowski, Malloy, Stegemann, Bush-Joseph, Harris and Nho. This is an open-access article distributed under the terms of the Creative Commons Attribution License (CC BY). The use, distribution or reproduction in other forums is permitted, provided the original author(s) or licensor are credited and that the original publication in this journal is cited, in accordance with accepted academic practice. No use, distribution or reproduction is permitted which does not comply with these terms. 